EUROPEAN JOURNAL OF PURE AND APPLIED MATHEMATICS

Vol. 12, No. 1, 2019, 14-24

ISSN 1307-5543 - www.ejpam.com

Published by New York Business Global

\title{
DG Poisson adjoint action and its application
}

\author{
Xiaojie $\mathrm{Li}^{1}$, Xianguo $\mathrm{Hu}^{1}$, Jiafeng $\mathrm{Lü}^{1}$, Xingting $\mathrm{Wang}^{2, *}$ \\ ${ }^{1}$ Department of Mathematics, Zhejiang Normal University, Jinhua, Zhejiang, 321004 \\ P.R. China \\ ${ }^{2}$ Department of Mathematics, Howard University, Washington DC, 20059, USA
}

\begin{abstract}
In this paper, the differential graded (DG for short) Poisson adjoint action on $M$ is introduced, where $M$ is a DG Poisson module over a DG Poisson Hopf algebra $A$. As an application, we give a new DG poisson module structure over the DG Poisson Hopf algebra $A$, which depends heavily on the structure of $A$.
\end{abstract}

2010 Mathematics Subject Classifications: 16E45, 16S10, 17B35, 17B63

Key Words and Phrases: Differential graded Poisson Hopf algebras, differential graded Poisson modules, the DG Poisson adjoint action

\section{Introduction}

The Poisson bracket was originally introduced by French Mathematician Siméon Denis Poisson in search for integrals of motion in Hamiltonian mechanics. Recently, different generalizations of Poisson algebras have been introduced by several people: Poisson orders [1], noncommutative Leibniz-Poisson algebras [2], Left-right noncommutative Poisson algebras [3], graded Poisson algebras [4], Poisson Ore-extensions [8], differential graded Poisson algebra [9], Poisson PI algebras [12], double Poisson algebras [19], Novikov-Poisson algebras [20] and Quiver Poisson algebras [22], etc. One of the most interesting features in this area is the Poisson universal enveloping algebra, which was first introduced by Oh [13] in order to describe the category of Poisson modules. Most recently, many people show their interests in Poisson universal enveloping algebras [8, 10, 16, 18, 21]. In particular, the second author of the present paper studied the universal enveloping algebras of DG Poisson algebras and propose a definition for the DG Poisson module [9].

We know that, Poisson Hopf algebras arise naturally in Poisson geometry and quantum groups. Recently, Poisson Hopf algebras are studied by many authors from different perspectives $[5,7,14,15]$. In [5], the authors developed the theory of Poisson Hopf algebras,

${ }^{*}$ Corresponding author.

DOI: https://doi.org/10.29020/nybg.ejpam.v12i1.3368

Email addresses: jiafenglv@zjnu.edu.cn (J.-F. Lü), 2015210420@zjnu.edu.cn (X.-G. Hu), 407586291@qq.com (X.-J. Li), xingting.wang@howard.edu (X. Wang) 
given the definition of a DG Poisson Hopf algebra $A$, and discussed the structures for the universal enveloping algebra of $A$.

Considering the importance of DG Poisson Hopf algebras and DG Poisson modules, our aim in this paper is to study the DG Poisson adjoint action on $M$, where $M$ is a DG Poisson modules over a DG Poisson Hopf algebras A. Furthermore, according to the DG Poisson adjoint action on $M$, we will construct a new DG Poisson module structure over $A$, which relies heavily on the structure of $A$.

The paper is organized as follows. In Section 2, we briefly review some basic definitions and results related to DG Poisson Hopf algebras and DG Poisson modules over DG Poisson algebras. In Section 3, we first propose a definition for the DG Poisson adjoint action, then discuss some basic properties of the DG Poisson adjoint action. As an application, we construct a new DG Poisson module structure over a DG Poisson Hopf algebra $A$.

Throughout the whole paper, $\mathbb{Z}$ denotes the set of integers, $\mathbb{k}$ denotes a base field of characteristic zero unless otherwise stated, and all (graded) algebras are assumed to have an identity and all (graded) modules are assumed to be unitary. We always take the grading to be $\mathbb{Z}$-graded. In addition, Let $V$ and $W$ be graded vector spaces, the twisting map $T: V \otimes W \rightarrow W \otimes V$ is defined for homogeneous elements $v \in V$ and $w \in W$ by

$$
T(v \otimes w)=(-1)^{|v||w|} w \otimes v
$$

and extends to all elements of $V$ and $W$ through linearity.

\section{Preliminaries}

In this section, we will recall some definitions and properties of DG Poisson Hopf algebras and DG Poisson modules.

By a graded algebra $A$ we mean a $\mathbb{Z}$-graded algebra $(A, u, \eta)$, where $u: A \otimes A \rightarrow A$ and $\eta: \mathbb{k} \rightarrow A$ are called the multiplication and unit of $A$, respectively. For convenience, we shall write $u(a \otimes b)$ as $a b, \forall a, b \in A$, whenever this does not cause confusion.

Recall that a graded coalgebra $C$ over $\mathbb{k}$ is a $\mathbb{Z}$-graded vector space with the graded linear maps $\Delta: C \rightarrow C \otimes C$ and $\varepsilon: C \rightarrow \mathbb{k}$ of degree 0 such that the obvious (usual) diagrams commute, where $\Delta$ and $\varepsilon$ are called the comultiplication and counit of $C$, respectively.

For any homogeneous element $c \in C$, we shall use the Sweedler's notation, that is $\Delta(c)=\sum_{(c)} c_{(1)} \otimes c_{(2)}$. In this notation, the comultiplication and counit property may be expressed as

$$
\begin{aligned}
(\Delta \otimes I) \Delta(c) & =(I \otimes \Delta) \Delta(c)=\sum_{(c)} c_{(1)} \otimes c_{(2)} \otimes c_{(3)}, \\
c & =\sum_{(c)} \varepsilon\left(c_{(1)}\right) c_{(2)}=\sum_{(c)} c_{(1)} \varepsilon\left(c_{(2)}\right)
\end{aligned}
$$

for any homogeneous elements $c \in C$, respectively.

Let $H$ be a graded algebra with multiplication $u$ and unit $\eta$, and at the same time a graded coalgebra with comultiplication $\Delta$ and counit $\varepsilon$. If $\Delta$ and $\varepsilon$ are graded algebra 
homomorphisms, then $H$ is called a graded bialgebra. Further, if $H$ admits a graded vector space homomorphism $S: H \rightarrow H$ of degree 0 which satisfies the following defining relation:

$$
u \circ(I \otimes S) \circ \Delta=u \circ(S \otimes I) \circ \Delta=\eta \circ \varepsilon .
$$

Then $H$ is called a graded Hopf algebra, and $S$ is called the antipode of $H$.

Similarly, a graded Poisson algebra can be defined as a graded algebra with a compatible graded Lie structure.

Definition 1. [4] Let $(A, \cdot)$ be a graded $\mathbb{k}$-algebra. If there is a $\mathbb{k}$-linear map

$$
\{\cdot, \cdot\}: A \otimes A \rightarrow A
$$

of degree 0 such that

(i) $(A,\{\cdot, \cdot\})$ is a graded Lie algebra. That is to say, we have

(ia) $\{a, b\}=-(-1)^{|a||b|}\{b, a\}$;

(ib) $\{a,\{b, c\}\}=\{\{a, b\}, c\}+(-1)^{|a||b|}\{b,\{a, c\}\}$,

(ii) (graded commutativity): $a \cdot b=(-1)^{|a||b|} b \cdot a$;

(iii) (biderivation property): $\{a, b \cdot c\}=\{a, b\} \cdot c+(-1)^{|a||b|} b \cdot\{a, c\}$,

for any homogeneous elements $a, b, c \in A$, then $A$ is called a graded Poisson algebra.

Definition 2. [5] Let $A$ be a graded $\mathbb{k}$-vector space. If there is a $\mathbb{k}$-linear map

$$
\{\cdot, \cdot\}: A \otimes A \rightarrow A
$$

of degree 0 such that:

(i) $(A, u, \eta,\{\cdot, \cdot\})$ is a graded Poisson algebra;

(ii) $(A, u, \eta, \Delta, \varepsilon)$ is a graded Hopf algebra;

(iii) $\Delta\left(\{a, b\}_{A}\right)=\{\Delta(a), \Delta(b)\}_{A \otimes A}$ for all $a, b \in A$, where the Poisson bracket $\{\cdot, \cdot\}_{A \otimes A}$ on $A \otimes A$ is defined by

$$
\left\{a \otimes a^{\prime}, b \otimes b^{\prime}\right\}_{A \otimes A}=(-1)^{\left|a^{\prime}\right||b|}\left(\{a, b\} \otimes a^{\prime} b^{\prime}+a b \otimes\left\{a^{\prime}, b^{\prime}\right\}\right)
$$

for any homogeneous elements $a, b, a^{\prime}, b^{\prime} \in A$.

Then $A$ is called a graded Poisson Hopf algebra. If in addition, there is a $\mathbb{k}$-linear homogeneous map $d: A \rightarrow A$ of degree 1 such that $d^{2}=0$ and

(iva) $d(\{a, b\})=\{d(a), b\}+(-1)^{|a|}\{a, d(b)\}$;

(ivb) $d(a \cdot b)=d(a) \cdot b+(-1)^{|a|} a \cdot d(b)$; 
(ivc) $\varepsilon \circ d=0$ and $\Delta d(a)=\sum_{(a)} d\left(a_{(1)}\right) \otimes a_{(2)}+\sum_{(a)}(-1)^{\left|a_{(1)}\right|} a_{(1)} \otimes d\left(a_{(2)}\right)$, where $\Delta(a)=\sum_{(a)} a_{(1)} \otimes a_{(2)}$,

for any homogeneous elements $a, b \in A$, then $A$ is called a DG Poisson Hopf algebra, which is usually denoted by

$$
(A, u, \eta, \Delta, \varepsilon, S,\{\cdot, \cdot\}, d) .
$$

Remark 1. By the formula (2.1) and $\Delta\left(\{a, b\}_{A}\right)=\{\Delta(a), \Delta(b)\}_{A \otimes A}$, we have

$$
\Delta(\{a, b\})=\sum_{(a)(b)}(-1)^{\left|a_{(2)}\right|\left|b_{(1)}\right|}\left(\left\{a_{(1)}, b_{(1)}\right\} \otimes a_{(2)} b_{(2)}+a_{(1)} b_{(1)} \otimes\left\{a_{(2)}, b_{(2)}\right\}\right)
$$

for all homogeneous elements a,b of a DG Poisson Hopf algebra A.

If $A$ is just a graded Hopf algebra, then the antipode $S$ has the following properties $[6,11,17]$.

Lemma 1. Let $A$ be a graded Hopf algebra and $S$ its antipode; then

(i) $S \circ u=u \circ T \circ(S \otimes S)$,

(ii) $S \circ \eta=\eta$,

(iii) $\varepsilon \circ S=\varepsilon$,

(iv) $T \circ(S \otimes S) \circ \Delta=\Delta \circ S$,

(v) if $A$ is graded commutative or graded cocommutative, then $S \circ S=I$,

where $I: A \rightarrow A$ is the identity morphism and $T: A \otimes A \rightarrow A \otimes A$ is the twisting morphism.

Lemma 2. [5] If $(A, u, \eta, \Delta, \varepsilon, S,\{\cdot, \cdot\}, d)$ is a DG Poisson Hopf algebra, then $d S=S d$, $S(\{a, b\})=(-1)^{|a||b|}\{S(b), S(a)\}$ and $\varepsilon(\{a, b\})=0$ for all $a, b \in A$.

Definition 3. [9] Let $\left(A, \cdot,\{\cdot, \cdot\}_{A}, d\right)$ be a DG Poisson algebra. We call a $\mathbb{Z}$-graded vector space

$$
M=\bigoplus_{i \in \mathbb{Z}} M^{i}
$$

a left DG Poisson module over A provided that the following conditions are satisfied:

(i) $(M, \cdot, \partial)$ is a left $D G$ module over the $D G$ algebra A. Equivalently,

(ia) there is a $\mathbb{k}$-bilinear function-- $-: A \otimes M \rightarrow M$ of degree 0 such that $M$ is a left graded module over $A$, i.e., $A^{i} \cdot M^{j} \subseteq M^{i+j}$ for all $i, j \in \mathbb{Z}$,

(ib) there is a $\mathbb{k}$-linear map $\partial: M \rightarrow M$ of degree 1 such that $\partial^{2}=0$ and $\partial(a \cdot m)=$ $d(a) \cdot m+(-1)^{|a|} a \cdot \partial(m)$ for all homogeneous elements $a \in A$ and $m \in M$. 
(ii) $(M, \cdot,\{\cdot, \cdot\})$ is a left $\mathbb{Z}$-graded Poisson module over the graded Poisson algebra A. That is to say, there is another bilinear bracket $\{\cdot, \cdot\}_{M}: A \otimes M \rightarrow M$ of degree 0 such that

(iia) $\{a, b \cdot m\}_{M}=\{a, b\}_{A} \cdot m+(-1)^{|a||b|} b \cdot\{a, m\}_{M}$,

(iib) $\{a \cdot b, m\}_{M}=a \cdot\{b, m\}_{M}+(-1)^{|a||b|} b \cdot\{a, m\}_{M}$ and

(iic) $\left\{a,\{b, m\}_{M}\right\}_{M}=\left\{\{a, b\}_{A}, m\right\}_{M}+(-1)^{|a||b|}\left\{b,\{a, m\}_{M}\right\}_{M}$

for all homogeneous elements $a, b \in A$ and $m \in M$.

(iii) The linear function $\partial$ is compatible with the bracket $\{-,-\}_{M}$. That is, we have $\partial\left(\{a, m\}_{M}\right)=\{d(a), m\}_{M}+(-1)^{|a|}\{a, \partial(m)\}_{M}$ for all homogeneous elements $a \in A$ and $m \in M$.

We usually call $\partial$ the differential of $M$ and use a quadruple $\left(M, \cdot,\{\cdot, \cdot\}_{M}, \partial\right)$ to denote a DG Poisson module.

\section{DG Poisson adjoint action and its application}

In this section, we define the DG Poisson adjoint action, and construct a new DG Poisson module over a DG Poisson Hopf algebra $A$ by studying the DG Poisson adjoint action, which will take advantage of the structure of a DG Poisson Hopf algebra.

We begin with the following definition.

Definition 4. Let $M$ be a DG Poisson module over a DG Poisson Hopf algebra

$$
(A, u, \eta, \Delta, \varepsilon, S, d)
$$

. Then the DG Poisson adjoint action on $M$ is defined by

$$
a d_{a}(z)=\sum_{(a)}(-1)^{\left|a_{(1)}\right|\left|a_{(2)}\right|} S\left(a_{(2)}\right)\left\{a_{(1)}, z\right\}, \quad a \in A, z \in M,
$$

where $\Delta(a)=\Sigma_{(a)} a_{(1)} \otimes a_{(2)}$

There exists a canonical DG Poisson adjoint action on $A$ given by

$$
a d_{a}(z)=\sum_{(a)}(-1)^{\left|a_{(1)}\right|\left|a_{(2)}\right|} S\left(a_{(2)}\right)\left\{a_{(1)}, z\right\}, \quad a, z \in A,
$$

since $A$ is a DG Poisson $A$-module with Poisson module structure $\{a, z\}_{A}$ and $a z$ is the multiplication in $A$ for all $a, z \in A$. Moreover, the canonical DG Poisson adjoint action on $A$ satisfies the following relation:

$$
a d_{a}(z y)=a d_{a}(z) \cdot y+(-1)^{|a||z|} z \cdot a d_{a}(y), \quad a, z, y \in A .
$$

In particular, if $|a|=1$, then the canonical DG Poisson adjoint action is a graded derivation on $A$. 
Lemma 3. Let $(A, u, \eta, \Delta, \varepsilon, S, d)$ be a DG Poisson Hopf algebra and let $M$ be a $D G$ Poisson A-module. Then

$$
a d_{a}(z)=-\sum_{(a)} a_{(1)}\left\{S\left(a_{(2)}\right), z\right\}
$$

where $a \in A$ and $z \in M$.

Proof. By Definition 3, it is easy to see that $\left\{1_{A}, z\right\}=0$, for any homogeneous element $z \in M$. Then for any homogeneous elements $a \in A, z \in M$, we have

$0=\left\{\varepsilon(a) 1_{A}, z\right\}=\left\{\sum_{(a)} a_{(1)} S\left(a_{(2)}\right), z\right\}=\sum_{(a)}\left(a_{(1)}\left\{S\left(a_{(2)}\right), z\right\}+(-1)^{\left|a_{(1)}\right|\left|a_{(2)}\right|} S\left(a_{(2)}\right)\left\{a_{(1)}, z\right\}\right)$.

Therefore

$$
a d_{a}(z)=-\sum_{(a)} a_{(1)}\left\{S\left(a_{(2)}\right), z\right\}
$$

Lemma 4. Let $(A, u, \eta, \Delta, \varepsilon, S, d)$ be a DG Poisson Hopf algebra and let $M$ be a $D G$ Poisson A-module. For any homogeneous elements $a, b \in A$, we have

$$
a d_{a b}=\varepsilon(a) a d_{b}+(-1)^{|a||b|} \varepsilon(b) a d_{a} .
$$

Proof. Since $\Delta(a b)=\sum_{(a)(b)}(-1)^{\left|a_{(2)}\right|\left|b_{(1)}\right|} a_{(1)} b_{(1)} \otimes a_{(2)} b_{(2)}$ and $M$ is a DG Poisson module over $A$, then for any homogeneous elements $a, b \in A, z \in M$, we have

$$
\begin{aligned}
a d_{a b}(z)= & \sum_{(a)(b)}(-1)^{\left|a_{(1)} b_{(1)}\right|\left|a_{(2)} b_{(2)}\right|+\left|a_{(2)}\right|\left|b_{(1)}\right|} S\left(a_{(2)} b_{(2)}\right)\left\{a_{(1)} b_{(1)}, z\right\} \\
= & \sum_{(a)(b)}(-1)^{\left|a_{(1)} b_{(1)}\right|\left|a_{(2)} b_{(2)}\right|+\left|a_{(2)}\right|\left|b_{(1)}\right|+\left|a_{(2)}\right|\left|b_{(2)}\right|} S\left(b_{(2)}\right) S\left(a_{(2)}\right)\left(a_{(1)}\left\{b_{(1)}, z\right\}\right. \\
& \left.+(-1)^{\left|a_{(1)}\right|\left|b_{(1)}\right|} b_{(1)}\left\{a_{(1)}, z\right\}\right) \\
= & \sum_{(a)(b)}(-1)^{\left|b_{(1)}\right|\left|b_{(2)}\right|} a_{(1)} S\left(a_{(2)}\right) S\left(b_{(2)}\right)\left\{b_{(1)}, z\right\} \\
& +(-1)^{|a||b|} \sum_{(a)(b)}(-1)^{\left|a_{(1)}\right|\left|a_{(2)}\right|} b_{(1)} S\left(b_{(2)}\right) S\left(a_{(2)}\right)\left\{a_{(1)}, z\right\} \\
= & \varepsilon(a) a d_{b}(z)+(-1)^{|a||b|} \varepsilon(b) a d_{a}(z)
\end{aligned}
$$

by Lemma 1.

Lemma 5. Let $(A, u, \eta, \Delta, \varepsilon, S, d)$ be a DG Poisson Hopf algebra and let $M$ be a $D G$ Poisson A-module. For any homogeneous elements $a, b \in A$, we have

$$
a d_{\{a, b\}}=a d_{a} a d_{b}-(-1)^{|a||b|} a d_{b} a d_{a} .
$$


Proof. Since $\Delta(\{a, b\})=\sum_{(a)(b)}(-1)^{\left|a_{(2)}\right|\left|b_{(1)}\right|}\left(\left\{a_{(1)}, b_{(1)}\right\} \otimes a_{(2)} b_{(2)}+a_{(1)} b_{(1)} \otimes\left\{a_{(2)}, b_{(2)}\right\}\right)$ and $M$ is a DG Poisson module over $A$, then for any homogeneous elements $a, b \in A, z \in$ $M$, we have

$$
\begin{aligned}
& a d_{\{a, b\}}(z) \\
& =\sum_{(a)(b)}(-1)^{\left|\{a, b\}_{(1)} \|\{a, b\}_{(2)}\right|} S\left(\{a, b\}_{(2)}\right)\left\{\{a, b\}_{(1)}, z\right\} \\
& =\sum_{(a)(b)}(-1)^{\left(\left|a_{(1)}\right|+\left|b_{(1)}\right|\right)\left(\left|a_{(2)}\right|+\left|b_{(2)}\right|\right)+\left|a_{(2)}\right|\left|b_{(1)}\right|}\left(S\left(a_{(2)} b_{(2)}\right)\left\{\left\{a_{(1)}, b_{(1)}\right\}, z\right\}\right. \\
& \left.+S\left(\left\{a_{(2)}, b_{(2)}\right\}\right)\left\{a_{(1)} b_{(1)}, z\right\}\right) \\
& =\sum_{(a)(b)}(-1)^{\left|a_{(1)}\right|\left|a_{(2)} b_{(2)}\right|+\left|b_{(1)}\right|\left|b_{(2)}\right|+\left|a_{(2)}\right|\left|b_{(2)}\right|} S\left(b_{(2)}\right) S\left(a_{(2)}\right)\left[\left\{a_{(1)},\left\{b_{(1)}, z\right\}\right\}-(-1)^{\left|a_{(1)}\right|\left|b_{(1)}\right|}\left\{b_{(1)},\left\{a_{(1)}, z\right\}\right\}\right] \\
& +\sum_{(a)(b)}(-1)^{\left|a_{(1)}\right|\left|a_{(2)} b_{(2)}\right|+\left|b_{(1)}\right|\left|b_{(2)}\right|+\left|a_{(2)}\right|\left|b_{(2)}\right|}\left\{S\left(b_{(2)}\right), S\left(a_{(2)}\right)\right\}\left[a_{(1)}\left\{b_{(1)}, z\right\}+(-1)^{\left|a_{(1)}\right|\left|b_{(1)}\right|} b_{(1)}\left\{a_{(1)}, z\right\}\right]
\end{aligned}
$$

by Lemmas 1 and 2 .

But

$$
\begin{aligned}
& \sum_{(a)(b)}(-1)^{\left|a_{(1)}\right|\left|a_{(2)}\right|+\left|a_{(1)}\right|\left|b_{(2)}\right|+\left|b_{(1)}\right|\left|b_{(2)}\right|+\left|a_{(2)}\right|\left|b_{(2)}\right|}\left\{S\left(b_{(2)}\right), S\left(a_{(2)}\right)\right\} a_{(1)}\left\{b_{(1)}, z\right\} \\
= & \sum_{(a)(b)}-(-1)^{\left|a_{(1)}\right|\left|a_{(2)}\right|+\left|a_{(1)}\right|\left|b_{(2)}\right|+\left|b_{(1)}\right|\left|b_{(2)}\right|}\left\{S\left(a_{(2)}\right), S\left(b_{(2)}\right)\right\} a_{(1)}\left\{b_{(1)}, z\right\} \\
= & \sum_{(a)(b)}-(-1)^{\left|b_{(1)}\right|\left|b_{(2)}\right|} a_{(1)}\left[\left\{S\left(a_{(2)}\right), S\left(b_{(2)}\right)\left\{b_{(1)}, z\right\}\right\}-(-1)^{\left|a_{(2)}\right|\left|b_{(2)}\right|} S\left(b_{(2)}\right)\left\{S\left(a_{(2)}\right),\left\{b_{(1)}, z\right\}\right\}\right] \\
= & a d_{a} a d_{b}(z)+\sum_{(a)(b)}(-1)^{\left|b_{(1)}\right|\left|b_{(2)}\right|+\left|a_{(2)}\right|\left|b_{(2)}\right|} a_{(1)} S\left(b_{(2)}\right)\left\{S\left(a_{(2)}\right),\left\{b_{(1)}, z\right\}\right\}
\end{aligned}
$$

by Lemma 3.

Similarly, we have

$$
\begin{aligned}
& \sum_{(a)(b)}(-1)^{\left|a_{(1)}\right|\left|a_{(2)}\right|+\left|a_{(1)}\right|\left|b_{(2)}\right|+\left|b_{(1)}\right|\left|b b_{(2)}\right|+\left|a_{(2)}\right|\left|b_{(2)}\right|+\left|a_{(1)}\right|\left|b_{(1)}\right|}\left\{S\left(b_{(2)}\right), S\left(a_{(2)}\right)\right\} b_{(1)}\left\{a_{(1)}, z\right\} \\
= & -(-1)^{|a||b|} a d_{b} a d_{a}(z)-\sum_{(a)(b)}(-1)^{|a||| b|+| a_{(1)}|| a_{(2)}|+| a_{(2)}|| b_{(2)} \mid} b_{(1)} S\left(a_{(2)}\right)\left\{S\left(b_{(2)}\right),\left\{a_{(1)}, z\right\}\right\} .
\end{aligned}
$$

Since for any homogeneous element $z \in M$, we have $\left\{1_{A}, z\right\}=0$. Thus

$$
\begin{aligned}
& a d_{\{a, b\}}(z) \\
= & a d_{a} a d_{b}(z)+\sum_{(a)(b)}(-1)^{\left|b_{(1)}\right|\left|b_{(2)}\right|+\left|a_{(2)}\right|\left|b_{(2)}\right|} a_{(1)} S\left(b_{(2)}\right)\left\{S\left(a_{(2)}\right),\left\{b_{(1)}, z\right\}\right\} \\
& -(-1)^{|a||b|} a d_{b} a d_{a}(z)-\sum_{(a)(b)}(-1)^{|a||b|+\left|a_{(1)}\right|\left|a_{(2)}\right|+\left|a_{(2)}\right|\left|b_{(2)}\right|} b_{(1)} S\left(a_{(2)}\right)\left\{S\left(b_{(2)}\right),\left\{a_{(1)}, z\right\}\right\}
\end{aligned}
$$




$$
\begin{aligned}
& +\sum_{(a)(b)}(-1)^{\left|a_{(1)}\right|\left|a_{(2)}\right|+\left|a_{(1)}\right|\left|b_{(2)}\right|+\left|b_{(1)}\right|\left|b_{(2)}\right|+\left|a_{(2)}\right|\left|b_{(2)}\right|} S\left(b_{(2)}\right) S\left(a_{(2)}\right)\left\{a_{(1)},\left\{b_{(1)}, z\right\}\right\} \\
& -\sum_{(a)(b)}(-1)^{\left|a_{(1)}\right|\left|a_{(2)}\right|+\left|a_{(1)}\right|\left|b_{(2)}\right|+\left|b_{(1)}\right|\left|b_{(2)}\right|+\left|a_{(2)}\right|\left|b_{(2)}\right|+\left|a_{(1)}\right|\left|b_{(1)}\right|} S\left(b_{(2)}\right) S\left(a_{(2)}\right)\left\{b_{(1)},\left\{a_{(1)}, z\right\}\right\} \\
= & a d_{a} a d_{b}(z)-(-1)^{|a||b|} a d_{b} a d_{a}(z)+\sum_{(b)}(-1)^{\left|b_{(1)}\right|\left|b_{(2)}\right|+|a|\left|b_{(2)}\right|} S\left(b_{(2)}\right)\left\{\varepsilon(a) 1_{A},\left\{b_{(1)}, z\right\}\right\} \\
& -\sum_{(a)}(-1)^{|a||b|+\left|a_{(1)}\right|\left|a_{(2)}\right|+\left|a_{(2)}\right||b|} S\left(a_{(2)}\right)\left\{\varepsilon(b) 1_{A},\left\{a_{(1)}, z\right\}\right\} \\
= & a d_{a} a d_{b}(z)-(-1)^{|a||b|} a d_{b} a d_{a}(z) .
\end{aligned}
$$

As an application, we are ready to state and prove our main result.

Theorem 1. Let $M$ be a DG Poisson module over a DG Poisson Hopf algebra

$$
(A, u, \eta, \Delta, \varepsilon, S, d)
$$

. Define

$$
\begin{array}{ll}
\alpha: A \times M \rightarrow M, & (a, z) \mapsto a \circ z=\varepsilon(a) z ; \\
\beta: A \times M \rightarrow M, & (a, z) \mapsto a * z=a d_{a}(z):=\sum_{(a)}(-1)^{\left|a_{(1)}\right|\left|a_{(2)}\right|} S\left(a_{(2)}\right)\left\{a_{(1)}, z\right\} .
\end{array}
$$

Then $(M, \circ, *, \partial)$ is a $D G$ Poisson A-module.

Proof. Note that $(A, u, \eta, \Delta, \varepsilon, S, d)$ is a DG Poisson Hopf algebra, we have that $\varepsilon$ is a graded algebra homomorphism. The fact $(M, \circ)$ is a left $\mathbb{Z}$-graded module over $A$ is straightforward and follows easily from the Definition of a graded module.

Now, let us prove that $(M, \circ, *)$ is a left $\mathbb{Z}$-graded Poisson module over the graded Poisson algebra $A$. By Lemma 2 , we know that $\varepsilon(\{a, b\})=0, \forall a, b \in A$. Note that $\mathbb{k}$ is a trivial DG Poisson Hopf algebra concentrated in degree 0 with trivial Poisson bracket and trivial differential, hence

$\{a, b\} \circ m+(-1)^{|a||b|} b \circ(a * m)=\varepsilon(\{a, b\}) m+(-1)^{|a||b|} \varepsilon(b) a d_{a}(m)=\varepsilon(b) a d_{a}(m)=a *(b \circ m)$.

For any homogeneous elements $a, b \in A, m \in M$, we have

$(a b) * m=a d_{a b}(m)=\varepsilon(a) a d_{b}(m)+(-1)^{|a||b|} \varepsilon(b) a d_{a}(m)=a \circ(b * m)+(-1)^{|a||b|} b \circ(a * m)$

and

$\{a, b\} * m=a d_{\{a, b\}}(m)=a d_{a} a d_{b}(m)-(-1)^{|a||b|} a d_{b} a d_{a}(m)=a *(b * m)-(-1)^{|a||b|} b *(a * m)$

by Lemmas 4 and 5 . Thus $(M, \circ, *)$ is a left $\mathbb{Z}$-graded Poisson module over $A$. 
Finally, we show that the differential $\partial$ satisfies the corresponding conditions. For any homogeneous elements $a \in A, m \in M$, we have on one hand:

$$
d(a) \circ m+(-1)^{|a|} a \circ \partial(m)=\varepsilon(d(a)) m+(-1)^{|a|} \varepsilon(a) \partial(m)=\varepsilon(a) \partial(m)=\partial(a \circ m),
$$

since $\varepsilon d=0$ and $\mathbb{k}$ is a trivial DG Poisson Hopf algebra concentrated in degree 0; and on the other hand:

$$
\begin{aligned}
\partial(a * m)= & \partial\left(a d_{a}(m)\right)=\partial\left(\sum_{(a)}(-1)^{\left|a_{(1)}\right|\left|a_{(2)}\right|} S\left(a_{(2)}\right)\left\{a_{(1)}, m\right\}\right) \\
= & \sum_{(a)}(-1)^{\left|a_{(1)}\right|\left|a_{(2)}\right|}\left(d S\left(a_{(2)}\right)\left\{a_{(1)}, m\right\}+(-1)^{\left|a_{(2)}\right|} S\left(a_{(2)}\right) \partial\left(\left\{a_{(1)}, m\right\}\right)\right) \\
= & \sum_{(a)}(-1)^{\left|a_{(1)}\right|\left|a_{(2)}\right|} S d\left(a_{(2)}\right)\left\{a_{(1)}, m\right\} \\
& +\sum_{(a)}(-1)^{\left|a_{(1)}\right|\left|a_{(2)}\right|+\left|a_{(2)}\right|} S\left(a_{(2)}\right)\left[\left\{d\left(a_{(1)}\right), m\right\}+(-1)^{\left|a_{(1)}\right|}\left\{a_{(1)}, \partial(m)\right\}\right], \\
= & a d_{d(a)}(m)+(-1)^{|a|} a d_{a}(\partial(m)) \\
= & \sum_{(a)}(-1)^{\left|d\left(a_{(1)}\right)\right|\left|a_{(2)}\right|} S\left(a_{(2)}\right)\left\{d\left(a_{(1)}\right), m\right\}+\sum_{(a)}(-1)^{\left|a_{(1)}\right|\left|d\left(a_{(2)}\right)\right|+|| a_{(1)}||} S d\left(a_{(2)}\right)\left\{a_{(1)}, m\right\} \\
& +(-1)^{|a|}\left(\sum_{(a)}(-1)^{\left|a_{(1)}\right|\left|a_{(2)}\right|} S\left(a_{(2)}\right)\left\{a_{(1)}, \partial(m)\right\}\right)
\end{aligned}
$$

by Lemma 2 and the following identity:

$$
\Delta d(a)=d\left(a_{(1)}\right) \otimes a_{(2)}+(-1)^{\left|a_{(1)}\right|} a_{(1)} \otimes d\left(a_{(2)}\right) .
$$

Hence

$$
\partial(a * m)=d(a) * m+(-1)^{|a|} a * \partial(m) .
$$

Therefore $(M, \circ, *, \partial)$ is a DG Poisson $A$-module.

\section{References}

[1] K. A. Brown and I. Gordon, Poisson orders, symplectic reflection algebras and representation theory, J. Reine Angew. Math. 559 (2003), 193-216.

[2] J. M. Casas and T. Datuashvili, Noncommutative Leibniz-Poisson algebras, Comm. Algebra 34(7) (2006), 2507-2530.

[3] J. M. Casas, T. Datuashvili and M. Ladra, Left-right noncommutative Poisson algebras, Cent. Eur. J. Math. 12(1) (2014), 57-78. 
[4] A. S. Cattaneo, D. Fiorenza and R. Longoni, Graded Poisson algebras, Encyclopedia Math. Phy. (2006), 560-567.

[5] M.-T. Guo, X.-G. Hu, J.-F. Lü and X.-T. Wang, The structures on the universal enveloping algebras of differential graded Poisson Hopf algebras, Comm. Algebra 46(6) (2018), 2714-2729.

[6] M. D. Gould, R. B. Zhang and A. J. Bracken, Quantum double construction for graded Hopf algebras, J. Bull. Austral. Math. Soc. 47 (1993), 353-375.

[7] J.-F. Lü, X. Wang and G.-B. Zhuang, Universal enveloping algebras of Poisson Hopf algebras, J. Algebra 426 (2015), 92-136.

[8] J.-F. Lü, X. Wang and G.-B. Zhuang, Universal enveloping algebras of Poisson Oreextensions, Proc. Amer. Math. Soc. 143 (2015), 4633-4645.

[9] J.-F. Lü, X. Wang and G.-B. Zhuang, DG Poisson algebra and its universal enveloping algebra, Sci. China Math. 59 (2016), 849-860.

[10] J.-F. Lü, S.-Q. Oh, X. Wang and X.-L. Yu, Enveloping algebras of double Poisson-Ore extensions, Comm. Algebra (2018) https://doi.org/10.1080/00927872.2018.1459643.

[11] J. M. Milner and J. C. More, On the structure of Hopf algebras, Ann. Math. 81 (1965), 211-264.

[12] S. P. Mishchenko, V. M. Petrogradsky and A. Regev, Poisson PI algebras, Trans. Amer. Math. Soc. 359(10) (2007), 4669-4694.

[13] S.-Q. Oh, Poisson enveloping algebras, Comm. Algebra 27 (1999), 2181-2186.

[14] S.-Q. Oh, Hopf structure for Poisson enveloping algebras, Beitrage Zur Algebra Und Geometrie. 44(44) (2003), 567-574.

[15] S.-Q. Oh, Poisson Hopf algebra related to a twisted quantum group, Comm. Algebra 45(1) (2016), 76-104.

[16] S.-Q. Oh, C.-G Park and Y.-Y Shin, A Poincaré-Birkhoff-Witt theorem for Poisson enveloping algebras, Comm. Algebra 30(10) (2002), 4867-4887.

[17] M. E. Sweedler, Hopf algebras, Benjamin, New York, 1969.

[18] U. Umirbaev, Universal enveloping algebras and universal derivations of Poisson algebras, J. Algebra 354 (2012), 77-94.

[19] M. Van den Berger, Double Poisson algebras, Trans. Amer. Math. Soc. 360(11) (2008), $5711-5769$.

[20] X.-P. Xu, Novikov-Poisson algebras, J. Algebra 190(2) (1997), 253-279. 
[21] Y.-H. Yang, Y. Yao and Y. Ye, (Quasi-)Poisson enveloping algebras, Acta Math. Sin. (Engl. Ser.) 29(1) (2013), 105-118.

[22] Y. Yao, Y. Ye and P. Zhang, Quiver Poisson algebras, J. Algebra 312(2) (2007), $570-589$. 